\title{
EFEITOS DA ANGIOTENSINA II NO SISTEMA CARDIOVASCULAR
}

\section{Effects of angiotensin II in the vascular system}

\author{
TRAPP, S.M. ${ }^{1}$; VAILATI, M.C.F. ${ }^{2}$; MATSUBARA, B.B. ${ }^{3}$; SCHWARTZ, D.S. ${ }^{2}$ \\ 1 Universidade Norte do Paraná, UNOPAR, Arapongas/PR. \\ ${ }^{2}$ Faculdade de Medicina Veterinária e Zootecnia, UNESP, Campus de Botucatu/SP. \\ ${ }^{3}$ Faculdade de Medicina, UNESP, Campus de Botucatu/SP.
}

Endereço para correspondência: Maria do Carmo Fernandez Vailati: mfvailati@yahoo.com.br

\section{RESUMO}

A angiotensina II é um importante peptídeo do sistema renina-angiotensina-aldosterona (SRAA). Esse peptídeo tem um papel importante na regulação da pressão sanguínea e homeostase dos fluidos corporais. Contudo, a sua atuação em condições anormais gera efeitos deletérios ao sistema cardiovascular. Resistência vascular, hipertensão, hipertrofia de miócitos, estímulo à produção de radicais livres e substâncias pró-inflamatórias são algumas das ações da angiotensina II que podem resultar no evento denominado de remodelação cardiovascular. Inibidores da enzima conversora de angiotensina (ECA), antagonistas dos receptores de angiotensina, anti-inflamatórios e anti-oxidantes são utilizados clínica e/ou experimentalmente para prevenir ou reduzir os efeitos da angiotensina II. Este trabalho tem como objetivo rever na literatura as ações e interações do peptídeo angiotensina II no sistema cardiovascular. Assim como, as possibilidades terapêuticas empregadas para o controle dos seus efeitos.

Palavras-chave: angiotensina II; coração; hipertensão; vasos sanguíneos

\section{ABSTRACT}

Angiotensin is an important peptide of renin-angiotensin-aldosterone system. This peptide has an important function on arterial blood pressure regulation and body fluid homeostasis. However, its action on abnormal conditions causes deleterious effects on the cardiovascular system. Vascular resistance, hypertension, vascular and myocytes hipertrophy, production of free radicals and pro-inflammatory substances are some of the actions of angiotensin II that can result on cardiovascular remodeling. Angiotensinconverting enzyme (ACE) inhibitors, angiotensin receptors antagonists, antiinflammatories and antioxidants are used clinically and/or experimentally to prevent or reduce the effects of angiotensin II. The purpose of this work is to review the actions and interactions of angiotensin II on the cardiovascular system, as well as the therapeutic measures employed for the control of these effects.

Key words: angiotensin II; blood vessels; heart; hypertension 


\section{INTRODUÇÃO}

As cardiopatias representam em torno de $11 \%$ das enfermidades apresentadas por cães (Buchanan, 1999). No Brasil, um estudo retrospectivo sobre 854 casos de cães, atendidos no Hospital Veterinário da Universidade de São Paulo, junto ao Serviço de Cardiologia, no período entre 1988 e 1992, revelou que as cardiopatias adquiridas representavam $52,4 \%$ do total, enquanto as cardiopatias congênitas, 3,63\% (Franken et al., 1994). O desfecho comum das cardiopatias é a falência ventricular e a manifestação clínica de insuficiência cardíaca (IC).

A insuficiência cardíaca é uma síndrome clínica complexa que pode resultar de qualquer doença cardíaca ou metabólica crônica que prejudica a habilidade dos ventrículos em ejetar sangue. Os eventos compensatórios inicialmente preservam o débito cardíaco e a pressão sanguínea e posteriormente levam a deterioração progressiva da função do miocárdio (Carelock e Clark, 2001).

O Sistema Renina Angiotensina Aldosterona (SRAA) tem um papel central na homeostase cardiovascular sendo a angiotensina II (ANG II) o principal mediador de sua ação fisiológica. A ação direta da ANG II no miocárdio modula a função cardíaca, como evidenciada pelos efeitos na contratilidade e no metabolismo do miocárdio além de permitir o desenvolvimento de alterações estruturais referentes à remodelação cardiovascular, como hipertrofia e fibrose miocárdica (Kjaer e Hesse, 2001). Indiretamente, a ANG II é capaz de regular a produção da citocina TNF- $\square$, associada a efeitos deletérios na função cardíaca (TorreAmione et al., 1996; Libera et al., 2001).

Portanto, por mecanismos diferentes, a ANG II participa da homeostase e da fisiopatologia cardiovascular. Considerando isto, a busca constante de um entendimento cada vez maior das ações da ANG II, contribuirá com os estudos relacionados à patogenia da IC.

\section{DESENVOLVIMENTO}

\section{o Sistema renina-angiotensina- aldosterona (SRAA)}

$\mathrm{Na}$ década de 50, foram reconhecidas duas formas de angiotensina, sendo a primeira um decapeptídeo (angiotensina I) e a segunda um octapeptídeo (angiotensina II). A formação da ANG II é decorrente da clivagem enzimática da angiotensina I (ANG I) por uma enzima peptidil-dipeptidase denominada enzima conversora de angiotensina (ECA). Posteriormente, o sistema reninaangiotensina foi reconhecido como um sistema que estimula a síntese e a secreção de aldosterona e como um importante mecanismo fisiológico na regulação homeostática da pressão arterial e na composição eletrolítica dos líquidos corporais (Gross, 1968).

No início da década de 70, foram descobertos polipeptídeos que inibiam a formação de ANG II ou bloqueavam os receptores da ANG II. Estes peptídeos inibidores revelaram importantes papéis fisiológicos e patológicos do SRAA. Esses achados inspiraram o desenvolvimento de uma classe nova e muito eficaz de agentes anti-hipertensivos, os inibidores da ECA. Posteriormente, estudos experimentais e clínicos realizados com inibidores da ECA, revelaram papéis adicionais para o SRAA na fisiopatologia da hipertensão, IC, vasculopatia, insuficiência renal, fibrose hepática e pulmonar, entre outros (Marshall et al., 2004). Desde então vários estudos foram realizados com o objetivo de desenvolver substâncias que inibem o SRAA ou os receptores da ANG II (Vailati, 2001; Joshi et al., 2005). 


\section{Componentes e ativação do SRAA}

Alguns tecidos, como o cérebro, hipófise, vasos sanguíneos, coração, rins e glândulas adrenais, expressam RNAm para renina, angiotensinogênio e/ou ECA. Entretanto, a importância fisiológica desse sistema renina-angiotensina intrínseco local ainda é pouco conhecida. Segundo Bader et al. (2001), há evidências de que os tecidos, anteriormente citados, contêm todos os componentes do SRAA e são, portanto, capazes de produzir ANG II local, entretanto, a contribuição desses sistemas locais para os níveis circulantes de angiotensinas ou reninas, ainda é controverso (Bregagnollo et al., 2005).

A quantidade de renina liberada é a principal determinante da taxa de produção de ANG II. A renina é sintetizada, armazenada e secretada na circulação arterial renal pelas células justaglomerulares situadas nas paredes das arteríolas aferentes dos néfrons (Silverthorn, 2003).

O angiotensinogênio é uma a2globulina, produzida no fígado, que circula em quantidades abundantes no plasma e é utilizada como substrato para a renina. A porção importante do angiotensinogênio é o terminal amina, a partir do qual a ANG I é clivada pela renina (Cassis et al., 1988).

A ECA plasmática e endotelial pulmonar converte a ANG I circulante em ANG II, por isso é considerada como uma peça importante do SRAA. Sabe-se que a ECA existe na face luminal das células endoteliais vasculares por toda a circulação e que a renina renal também pode ser captada pela parede arterial. Assim, a conversão do angiotensinogênio em ANG I, e a conversão de ANG I em ANG II ocorrem basicamente dentro, ou na superfície da parede do vaso sanguíneo (Silverthorn, 2003).

A carboxipeptidase relacionada à enzima conversora de angiotensina (ECA2) é uma enzima homóloga à ECA que se expressa predominantemente nas células endoteliais do coração, rins e testículos (Donoghue et al., 2000). A ECA2 é responsável por formar a angiotensina(1-7) por meio da clivagem da ANG II (Vickers et al., 2002). Contudo, são apresentadas evidências contraditórias quanto à formação da angiotensina-(1-7) a partir da clivagem da ANG I pela ECA2 (Donoghue et al., 2000; Campbell et al., 2004; Ferrario, 2006).

Ao contrário da ECA, a capacidade de clivagem do seu homólogo não é bloqueada com 0 uso de fármacos inibidores da ECA (Donoghue et al., 2000). Os estudos crescentes sobre a fisiologia $\mathrm{e}$ fisiopatologia da ECA2, ainda são pouco esclarecedores (Vickers et al., 2002), sugerindo que esta enzima seja responsável por equilibrar os efeitos vasoconstritores e vasodilatadores do SRAA a partir da redução na concentração de ANG II e, possivelmente, por contribuir para a formação de angiotensina-(1-7) (Santos et al., 2003; Ferrario 2006).

Pela ação de aminopeptidase, endopeptidases e da ECA há a formação de [des-Asp "1] ANG I, ANG II, ANG III e ANG IV. Apesar dos seus efeitos qualitativos serem semelhantes, a potência destes efeitos é menor quando comparada à potência dos efeitos da ANG II (Nishimura, 2001).

De forma direta ou indireta a ANG I e a ANG II também são metabolizadas em angiotensina-(1-7), a qual possui ações opostas às da ANG II. A angiotensina-(1-7) apresenta um efeito potente na distribuição do fluxo sanguíneo e promovem alterações bioquímicas e funcionais que, além da vasodilatação, provocam a redução ou prevenção da remodelação cardíaca por meio da diminuição da hipertrofia cardíaca e da fibrose (Ferreira et al., 2001; Grobe et al., 2006; Santos e Ferreira, 2007). Os efeitos cardiovasculares benéficos deste heptapeptídeo parecem ser resultantes da regulação da ANG II ou da potencialização dos efeitos da bradicinina ou da liberação 
do óxido nítrico (Almeida et al., 2000; Sampaio et al., 2007).

A clivagem da ANG I pela ECA2 resulta na formação da angiotensina-(1-9), cuja função ainda é desconhecida (Donoghue et al., 2000).

Os efeitos das angiotensinas são exercidos por meio de receptores específicos da superfície celular. Em 1989, Chiu et al., caracterizaram dois subtipos de receptores da ANG II posteriormente definidos como $\mathrm{AT}_{1}$ e $\mathrm{AT}_{2}$. Até o momento, a maioria dos efeitos da angiotensina II parece ser mediada pelo receptor $\mathrm{AT}_{1}$ (Rajagopal et al., 2005). Acreditava-se que os efeitos dos receptores $A T_{1}$ e $A T_{2}$ eram opostos (Carey e Siragy, 2003), entretanto recentemente observou-se que o receptor $\mathrm{AT}_{2}$ também é capaz de atuar de forma semelhante ao receptor $A T_{1}$ no que se refere às ações vasoconstritoras e proliferativas da ANG II (D'Amore et al., 2005).

As ações vasodilatadoras e antiproliferativas da angiotensina-(1-7) são, em parte, mediadas por receptores conhecidos como AT 1-7 Mas (Santos et al., 2003). Contudo, evidências apontam para a interação do receptor Mas da angiotensina-(1-7) com os receptores $A T_{1}$ e $\mathrm{AT}_{2}$ e para a existência de outros possíveis receptores (Castro et al., 2005).

\section{Ações e interações da ANG II no sistema cardiovascular}

Classicamente, a ANG II é conhecida por causar aumento direto e indireto da pressão arterial (PA), o qual é dosedependente (Li et al., 1998). A ANG II pode aumentar 0 volume circulatório estimulando a sede e a secreção de vasopressina $(A D H)$ e de aldosterona. $O$ volume circulatório aumentado associado a um maior estímulo simpático proporciona a elevação do débito cardíaco que, acrescido da ação vasoconstritora provocada pela ANG II elevam a PA (Silverthorn, 2003).
Pacientes com função cardíaca comprometida apresentam diminuição da PA e do fluxo plasmático renal, consequentemente ocorre a ativação do SRAA e a elevação dos níveis circulantes de ANG II para que haja o restabelecimento da PA. No entanto, a permanência desta condição provoca a deterioração do tecido cardíaco (Zisman, 2005).

A ANG II estimula a liberação e expressão do vasoconstritor endotelina pelas células endoteliais. $O$ aumento da pressão sanguínea e a hipertrofia vascular induzida pela ANG II é, em parte, induzida por um aumento na produção endógena de endotelinas, as quais ativam os receptores $\mathrm{ET}_{\mathrm{A}}$ a produzirem alterações no sistema cardiovascular (Moreau et al., 1997). A possibilidade de prevenir alterações cardiovasculares com o bloqueio dos receptores $\mathrm{ET}_{\mathrm{A}}$, sugere outra alternativa terapêutica nas doenças cardiovasculares associadas com o aumento da atividade do SRAA (Rajagopalan et al., 1997).

Estudos mais recentes enfatizaram a busca por outras formas de interferência da ANG II na PA. Há evidências que a ANG II é capaz de induzir resposta inflamatória na parede vascular por meio de mecanismos dependentes e independentes da pressão. A ANG II induz interações entre os leucócitos e o endotélio, aumentando a quimiotaxia com posterior elevação da produção de citocinas, incluindo IL-1, IL-6 e TNF - $\alpha$ (Cheng et al., 2005).

A ANG II também apresenta efeito na produção de radicais livres que geram a reação inflamatória vascular. A enzima NADPH oxidase, encontrada nas células da musculatura lisa vascular, é uma importante fonte de radicais livres e representa a principal produtora de ânions superóxido $\left(\mathrm{O}_{2}^{-}\right)$na hipertensão (Zimmerman et al., 2004; Cheng et al., 2005).

O uso de fármacos que inibem seletivamente a via cicloxigenase-2, como 
- rofecoxib, reduz a produção do $\mathrm{O}_{2}$, gerados pela enzima NADPH oxidase, reduzindo a hipertensão e a hipertrofia cardíaca em ratos. Desta forma, a via cicloxigenase-2 parece também participar do estresse oxidativo e dos efeitos deletérios ao sistema cardiovascular (Wu et al., 2005).

A proliferação das células da musculatura lisa vascular é um evento crucial no desenvolvimento de hipertensão e é ativado por vários fatores de crescimento e citocinas. Tem sido mostrado que a ANG II atua não somente como um peptídeo vasoativo, mas também como um fator de crescimento. Em particular, este estímulo proliferativo e hipertrófico nas células vasculares da musculatura lisa ocorre por meio da ativação do receptor $\mathrm{AT}_{1}$ (Li et al., 2005).

Em cultura de células da musculatura lisa vascular de ratos, o anti-oxidante ácido cafeíco diminuiu os níveis intracelulares do $\mathrm{O}_{2}^{-}$e a proliferação celular induzida por ANG II. A inibição da proliferação pode ser favorável, retardando o desenvolvimento da hipertensão e constituindo mais uma opção terapêutica nas doenças vasculares (Li et al., 2005).

A ANG II tem efeitos diretos e indiretos sobre o coração, os quais afetam a frequência cardíaca, a contratilidade e o crescimento celular. Induz à resposta inotrópica positiva, independente do sistema $\beta$-adrenérgico em várias espécies, exceto em ratos. Essa ação inotrópica é dose-dependente e mediada por receptores_de angiotensina (Baker et al., 1992). Indiretamente, a ANG II aumenta a frequência cardíaca em decorrência da facilitação do tônus simpático, do aumento da neurotransmissão noradrenérgica e da liberação de catecolaminas pela adrenal. Contudo, em situações de hipertensão com estimulação dos barorreceptores, a ANG II é capaz de diminuir a frequência cardíaca (Hu et al., 1998).

Cronicamente, a ANG II induz mudança na forma, composição e tamanho do coração, num processo denominado remodelação miocárdica. Essas alterações incluem hipertrofia, acúmulo de colágeno no compartimento intersticial com evolução para morte celular miocárdica e fibrose cardíaca (Sutton e Sharpe, 2000).

De acordo com o ciclo celular, os miócitos cardíacos são células permanentes, ou seja, incapazes de sofrer divisão, ficando a síntese protéica reduzida a taxas apropriadas para manutenção e reparação celular. Em condições de agressão, ocorre um aumento na síntese protéica com permanente bloqueio do ciclo celular resultando na hipertrofia anormal (Katz, 1994).

Apesar de a remodelação miocárdica ser considerada um mecanismo adaptativo, no sentido de preservar a função ventricular, o processo pode tornarse deletério quando 0 estímulo desencadeante é patológico e contínuo. Nestas condições a remodelação miocárdica contínua, habitualmente evolui, em tempo variável, em disfunção ventricular progressiva, que culmina com insuficiência cardíaca e morte prematura (Bregagnollo et al., 2005).

A participação da ANG II na remodelação do miocárdio é complexa, visto as diversas possibilidades que este peptídeo utiliza para tal evento. Muitos estudos têm sido realizados em busca de elementos relacionados a ANG II e que, de uma forma ou outra, contribuem com o mecanismo de remodelação. Mesmo assim, as pesquisas sugerem que ainda há muito por se descobrir.

A ANG II pode culminar na hipertrofia secundária ao aumento da pós-carga. Entretanto, este peptídeo também exerce ação trófica, independente da PA e resistência vascular, contribuindo para a remodelação miocárdica e consequentemente disfunção cardíaca (Daigle et al., 2004).

Efeitos proliferativos e hipertróficos associados à produção de radicais livres são induzidos pela ANG II. Indiretamente 
este peptídeo é capaz de induzir a transferência de sinais mitogênicos ao núcleo, resultando na expressão de genes de crescimento e acúmulo de proteínas no interior da célula (Li et al., 2005).

Os efeitos promotores de crescimento induzidos pela ANG II são principalmente mediados por fatores denominados como fator de crescimento dos fibroblastos (bFGF), fator de crescimento derivado das plaquetas (PDGF), fator de crescimento endotelial vascular (VEGF), fator de crescimento transformante de fibroblastos do tipo beta (TGF- $\beta$ ) e fator de crescimento do tecido conjuntivo (CTGF) (Cheng et al., 2005).

A necrose do miocárdio é mais um fenômeno responsável pelas alterações estruturais do coração. Experimentalmente, a ANG II tem sido associada à necrose dos miócitos cardíacos. Entretanto, é possível que este efeito também seja consequente da isquemia sofrida pelas fibras cardíacas (Okoshi et al., 1997).

Reforçando o conceito de "substância cardiotóxica", a ANG II foi associada a apoptose, outro mecanismo descrito como colaborador da remodelação. A exposição de células musculares à ANG II provoca apoptose, a qual é reduzida após o bloqueio dos receptores $\mathrm{AT}_{1}$ (Mascareno et al., 2005). Assim como nos vasos, em situações de agressão cardíaca provocada pela ANG II, também são identificadas citocinas inflamatórias teciduais e circulantes, em especial, o TNF- $\alpha$, via ativação do receptor $\mathrm{AT}_{1}$ (Mann, 2003).

O TNF- $\alpha$ atua por meio de dois receptores, os quais quando estimulados interagem com uma variedade de sinais. As ações do TNF- $\alpha$ dependem do tipo de estímulo o que justifica, em parte, as diferentes respostas observadas nas situações in vitro e in vivo e também as evidências conflitantes em relação às ações desta citocina na função e sobrevivência do cardiomiócito (Henriksen e Newby, 2003). Segundo Mann (2003), os efeitos benéficos e deletérios das citocinas podem ser influenciados também pelo tempo de expressão e pela concentração destas.

Há mais de uma década demonstrouse que pacientes com insuficiência cardíaca apresentam altos níveis séricos de TNF- $\alpha$ e que esses níveis são proporcionais à gravidade da doença (Torre-Amione et al., 1996).

Por ser relacionado à apoptose, à hipertrofia cardíaca e à depressão da função ventricular, o TNF- $\alpha$ também foi responsabilizado pela remodelação cardiovascular. A menor deposição de colágeno e a melhora na função diastólica em alguns estudos com a terapêutica antiTNF- $\alpha$ sustentam a hipótese do envolvimento desta citocina na remodelação (Li et al., 2000).

Exceto por apoptose, os mecanismos descritos anteriormente, se perpétuos, resultam na morte celular determinada ou acompanhada por inflamação. A morte celular é acompanhada por fibrose no tecido cardíaco, havendo um acúmulo de colágeno tipo-1. Assim, este tecido tornase ainda mais afuncional (Weber, 2004).

Embora a remodelação miocárdica seja um fenômeno complexo, envolvendo vários estímulos, o papel do SRAA ganhou especial evidência na última década, devido ao grande interesse por pesquisas que buscam interferir com o SRAA ( $\mathrm{Li}$ et al., 1998).

Anti-hipertensivos denominados inibidores da ECA bloqueiam a conversão mediada pela ECA de ANG I em ANG II, causam vasodilatação e reduzem a $P A$. Além disso, há menor liberação de aldosterona, diminuição na reabsorção de sódio e diminuição do fluido extracelular (Silverthorn, 2003). O inibidor da ECA captopril foi o primeiro anti-hipertensivo desenvolvido a partir das descobertas relacionadas à bradicinina (Ferreira, 2000).

Os inibidores da ECA ainda são amplamente utilizados não só com 0 objetivo de controlar a PA. O seu uso 
prolongado em pacientes com insuficiência cardíaca congestiva proporciona melhora dos índices hemodinâmicos, assim como dos sinais clínicos e da qualidade de vida (Hirakata et al., 1990).

Sartanas é outro grupo de fármaco que impede o aumento da PA decorrente dos efeitos da ANG II, do qual fazem parte os fármacos que bloqueiam os receptores $\mathrm{AT}_{1}$ e $\mathrm{AT}_{2}$. Inúmeros trabalhos têm mostrado que, principalmente, o bloqueio dos receptores $A T_{1}$ é capaz de reduzir a PA induzida por ANG II, amenizar danos aos órgãos-alvo e diminuir o risco de morte em pacientes com IC ( $\mathrm{Li}$ et al., 1998; Vailati, 2001).

Em estudos experimentais, os inibidores da ECA previnem a hipertrofia, a fibrose ventricular e a disfunção do ventrículo esquerdo (Bregagnollo et al., 2005). Evidências clínicas em seres humanos mostraram que os inibidores da ECA reduzem a morbidade e a mortalidade em pacientes com disfunção sistólica ventricular e com hipertensão (Boos, 2004).

Em medicina veterinária, diferentes estudos mostraram que cães com insuficiência cardíaca, causada por miocardiopatia dilatada ou regurgitação de mitral, tratados com enalapril apresentam melhora clínica. Os efeitos do fármaco em relação à sobrevida são menos conhecidos (Kittleson, 2004). Outras vantagens observadas na terapêutica com inibidores da ECA é a atenuação da apoptose em miócitos de cães com insuficiência cardíaca e a melhora da contração e do relaxamento de miócitos adjacentes às áreas de infarto (Kim et al., 2003).

Em relação ao bloqueio dos receptores de ANG II, observações experimentais identificaram que o bloqueio do receptor $\mathrm{AT}_{1}$ previne alterações estruturais e funcionais no ventrículo esquerdo (Li et al., 1998). A presença do $\mathrm{O}_{2}{ }^{-}$estimula indiretamente a ligação do receptor $A T_{1}$ à proteína $G$ induzindo $a$ proliferação das células da musculatura lisa vascular (Li et al., 2005).

Em seres humanos a inibição dos receptores $A T_{1}$ pelo valsartan reduz 0 número de hospitalizações e o risco de eventos cardiovasculares em maior proporção quando comparado ao uso de alguns inibidores da ECA como captopril e lisinopril (Pfeffer et al., 2003). A inibição dos receptores $\mathrm{AT}_{1}$ pelo candesartan também evidenciou a redução da morbidade e mortalidade em 15 pacientes com fração de ejeção do ventrículo esquerdo inferior a $40 \%$ (McMurray et al., 2003).

A terapêutica com valsartan em cães com insuficiência cardíaca reduz a pré e pós-carga e interfere de forma limitada na progressão da disfunção e remodelação do ventrículo esquerdo (Tanimura et al., 1999).

A literatura médica tem confirmado que os bloqueadores de angiotensina promovem proteção cardiovascular e são efetivos na redução da mortalidade cardiovascular. Além disso, relatos recentes têm sugerido que 0 uso combinado de bloqueadores do receptor de angiotensina $A T_{1}$ e de inibidores da ECA apresentam resultados superiores quando comparado ao uso isolado de uma destas substâncias (Eisenberg e Giola, 2006).

Considerando o envolvimento dos radicais livres e da inflamação, cresce o interesse por terapias antifilamatórias e anti-oxidantes como parte da prevenção da remodelação. Observações oriundas, principalmente, de modelos experimentais, têm estimulado pesquisas visando 0 potencial terapêutico dos anti-oxidantes, como o ácido caféico, antiiflamatórios e mais enfaticamente os medicamentos anti - TNF- $\alpha$ (Li et al., 2000; Li et al., 2005; Wu et al., 2005).

Nos últimos anos muito tem se explorado a respeito do bloqueio do TNF- $\alpha$ e diversos são os resultados encontrados. $O$ etanercept, um dos fármacos anti-TNF- $\alpha$ 
mais estudados, é um receptor do TNF- $\alpha$ tipo 2 solúvel que se liga ao TNF- $\alpha$ impedindo que este se ligue aos seus receptores presentes na superfície da membrana celular das células alvo (Mann, 2005).

São conflitantes as evidências clínicas observadas em pacientes com insuficiência cardíaca submetidos à terapia com etanercept. Alguns resultados demonstram que a terapia proporciona a redução dos níveis de TNF- $\alpha$, melhora na função ventricular e na condição clínica (Deswal, et al., 1999), enquanto outros demonstram um maior número de hospitalizações e maior risco de morte (Mann, 2005).

Pacientes humanos com insuficiência cardíaca tratados com infliximab, um anticorpo monoclonal que neutraliza 0 TNF- $\alpha$, também apresentaram maior risco de morte e maior número de hospitalizações quando comparados ao grupo controle (Chung et al., 2003). Um levantamento realizado por Kwon et al. (2003) junto ao U.S. Food and Drug Administration (FDA)'s Med Watch system constatou vários relatos de piora dos sinais clínicos em pacientes com insuficiência cardíaca submetidos à terapia com antagonistas do TNF- $\alpha$.

Segundo Mann (2003), dentre as possíveis justificativas para a piora dos pacientes tratados com anti- TNF- $\alpha$ é que esta citocina também possui efeitos fisiológicos que conferem proteção celular durante lesões isquêmicas agudas. Além disso, a ausência de melhores resultados também pode ser decorrente do envolvimento de outras citocinas que não são bloqueadas com a terapia anti TNF- $\alpha$.

\section{CONCLUSÕES}

Em condições de exacerbada e contínua ação da angiotensina II no sistema cardiovascular, várias alterações hemodinâmicas, bioquímicas e estruturais ocorrem com comprometimento da função cardíaca. A angiotensina II é clássicamente conhecida devido à sua potente ação vasoconstritora. Entretanto, independente da pressão, eventos hipertróficos, proliferativos, inflamatórios e oxidantes resultam na agressão e morte celular com evolução à fibrose do tecido (remodelação cardiovascular). Em decorrência dos seus efeitos cardiovasculares, este peptídeo é amplamente estudado. Novas descobertas em relação ao mecanismo de ação da ANG II têm proporcionado alterações na terapêutica atual. Porém, ainda são necessárias muitas pesquisas e estudos para uma maior elucidação de seu crescente e complexo mecanismo de ação.

\section{REFERÊNCIAS}

ALMEIDA, A.P.; FRÁBREGAS, B. C.; MADUREIRA, M. M. et al. Angiotensin-(1-7) potentiates the coronary vasodilatory effect of bradykinin in the isolated rat heart. Brazilian Journal of Medical and Biological Research, v.33, p.709-713, 2000.

BADER, M.; PETERS, J.; BALTATU, O. Tissue renin-angiotensin systems: new insights from experimental animal models in hypertension research. Journal of Molecular Medicine, v.79, p.76-102, 2001.

BAKER, K.M.; BOOZ, G.W.; DOSTAL, D.E. Cardiac actions of angiotensin II: role of an intracardiac renin-angiotensin system. Annual Review of Physiology, v.54, p.227-241, 1992.

BOOS, J. Cardiovascular protection with ace inhibitors - more HOPE for EUROPA? Medical Science Monitor, v.10, n.12, p.SR23-28, 2004.

BREGAGNOLLO, E.A.; OKOSHI, K.; BREGAGNOLLO, I.F. et al. Efeitos da inibição prolongada da enzima de conversão de angiotensina sobre as características morfológicas e funcionais da hipertrofia ventricular esquerda em ratos com sobrecarga pressórica persistente.

Arquivos Brasileiros de Cardiologia, v.84, n.3, p.225-32, 2005.

BUCHANAN, J.W. Prevalence of cardiovascular disorders. In: FOX, P.R.; SISSON, D. ; MOISES, N.S. Textbook of canine and feline cardiology: principles and clinical practice. 2. ed. Philadelphia: W.B. Saunders, 1999. Cap.23, p.457-470. 
CAMPBELL, D.J.; ZEITZ, C.J.; ESLER, M.D. et al. Evidence against role for angiotensin converting enzyme-related carboxypeptidase (ACE2) in angiotensin peptide metabolism in the human coronary circulation. Journal of Hypertension, v.22, n.10, p.1971-1976, 2004.

CARELOCK, J.; CLARK, A.P. Heart failure: pathophysiologic mechanisms: the same neurohormonal actions that initially preserve cardiac output subsequently cause functional deterioration. New drug breakthroughs may provide a solution. American Journal of Nursing, v.101, p.26-33, 2001.

CAREY, R.M.; SIRAGY, H.M. Newly recognized components of the renin-angiotensin sysrem: potential roles in cardiovascular and renal regulation. Endocrine Reviews, v.24, p.261-271, 2003.

CASSIS, L.A.; SAYE, J.; PEACH, M.J.. Location and regulation of rat angiotensinogen messenger RNA. Hypertension, v.11, p.591-596, 1988.

CASTRO, C.H.; SANTOS, R.A.; FERREIRA, A.J. et al. Evidence for a functional interaction of the angiotensin-(1-7) receptor Mas with AT1 and AT2 receptors in the mouse heart. Hypertension, v.46, part 2, p.937-942, 2005.

CHENG, Z.J.; VAPAATALO, H.; MERVAALA, E.. Angiotensin II and vascular inflammation. Medical Science Monitor, v.11, n.6, p.RA194-RA205, 2005.

CHIU, A.T.; HERBLIN, W.F.; MCCALL, D.E. et al. Identification of angiotensin II receptor subtypes.

Biochemical and Biophysical Research Communications, v.165, n.196-203, 1989.

CHUNG, E.S.; PACKER, M.; LO, K.H. et al. Randomized double-blind, placebo-controlled, pilot trial of infliximab, a chimeric monoclonal antibody to tumor necrosis factor - $\alpha$, in patients with moderateto-severe heart failure: results of the anti-TNF therapy against congestive heart failure $(\mathrm{ATTACH})$ trial. Circulation, v.107, p.3133-3140, 2003.

DAIGLE, C.; MARTENS, F.M.A.C.; GIRARDOT, D. et al. Signaling of angiotensin II-induced vascular protein synthesis in conduit and resistance arteries in vivo. BMC Cardiovascular Disorders, v.10, p.46, 2004.

D'AMORE, A.; BLACK, M.J.; THOMAS, W.G. The angiotensin 2 receptor causes constitutive growth of cardiomyocytes and does not antagonize angiotensin II type 1 receptor-mediated hypertrophy. Hypertension, v.46, n.6, p.1347-1354, 2005.

DESWAL, A.; BOZKURT, B.; SETA, Y. et al. Safety and efficacy of a soluble P75 tumor necrosis factor receptor (Enbrel, Etanercept) in patients with advanced heart failure. Circulation, v.99, p.32243226, 1999.

DONOGHUE, M.; HSIEH, F.; BARONAS, E. et al. A novel angiotensin-converting enzyme-related carboxypeptidase (ACE2) converts angiotensin I to angiotensin 1-9. Circulation Research, v.87, p.E1E9, 2000.

EISENBERG, M.J.; GIOLA, L.C. Angiotensin II receptor blockers in congestive heart failure. Cardiology Review, v.14, n.1, p.26-34, 2006.

FERRARIO, C.M. Angiotensin-converting enzyme 2 and angiotensin-(1-7) an envolving story in cardiovascular regulation. Hypertension, v.47, part 2, p.515-521, 2006.

FERREIRA, S.H. Angiotensin converting enzyme: history and relevance. Seminars in Perinatology, v.24, n.1, p.7-10, 2000.

FERREIRA, A.J.; SANTOS, R.A.; ALMEIDA, A.P.. Angiotensin-(1-7): cardioprotective effect in myocardial ischemia/reperfusion. Hypertension, v.38, part 2, p.665-668, 2001.

FRANKEN, A.; LARSSON, M.H.M.A.; PEREIRA, L. Estudo retrospectivo das cardiopatias diagnosticadas em cães / 1988 a 1992. In: Congresso Brasileiro de Medicina Veterinária; 23, 1994, Olinda, PE. Anais... Olinda: SBMV., 1994; p.414.

GROSS, F. The regulation of aldosterone secretion by the renin-angiotensin system under various conditions. Acta Endocrinologica, v.124, p.41-64, 1968.

GROBE, J.L.; MECCA, A.P; MAO, H. et al. Chronic angiotensin - (1-7) prevents cardiac fibrosis in DOCA-salt model of hypertension. American Journal of Physiology Heart and Circulatory Physiology, v.290, p.H2417-H2423, 2006.

HENRIKSEN, P.A.; NEWBY, D.E. Therapeutic inhibition of tumour necrosis factor $\alpha$ in patients with heart failure: cooling an inflamed heart. Heart, v.89, p.14-18, 2003.

HIRAKATA, H.; TARAZI, F.M.F.; BUMPUS, F.M. et al. Angiotensins and the failing heart. Circulation Research, v.66, p.891-899, 1990.

HU, L.; CATANZARO, D.F.; PITARRESI, T.M. et al. Identical hemodynamic and hormonal responses to 14-day infusions of rennin or angiotensin II in conscious rats. Journal of Hypertension, v.16, p.1285-1298, 1998.

JOSHI, S.R.; KALE, S.; OOMMAN, A. et al. Study of efficacy and safety of losartan (losar) in Asian Indian hypertensives. Journal of the Indian Medical Association, v.103, n.7, p.394-396, 2005. 
KATZ, A.M. The cardiomyopathy of overload: an unnatural grow response in the hypertrophied heart. Annals of Internal Medicine, v.121, p.363-371, 1994.

KIM, Y.; MANKAD, S.; KIM, S.J. et al. Adding angiotensin II type 1 receptor blockade to angiotensin-converting enzyme inhibition limits myocyte remodeling after myocardial infarction. Journal of Cardiac Failure, v.9, n.3, p.238-245, 2003.

KITTLESON, M.D. Terapia da insuficiência cardíaca. In: ETTINGER, S.J.; FELDMAN, E.C. Tratado de medicina interna veterinária: doenças do cão e do gato. 5 ed. Rio de Janeiro: Guanabara Koogan, 2004. Cap.111, p.762-764.

KJAER, A.; HESSE, B. Heart failure and neuroendocrine activation: diagnostic, prognostic and therapeutic perspectives. Clinical Physiology, v.6, p.661-672, 2001.

KWON, H. J.; COTÉ, T.R.; CUFFE, M.S. et al. Case reports of heart failure after therapy with a tumor necrosis factor antagonist. Annals of Internal Medicine, v.138, p.807-811, 2003.

LI, J.S. ; TOUYS, R.M.; SCHIFFRIN, E.L. Effects of AT1 and AT2 angiotensin receptor antagonists in angiotensin II-infused rats. Hypertension, v.31, n.2, p.487-492, 1998.

LI, Y.Y. ; FENG, Y.Q.; KADOKAMI, T. et al. Myocardial extracellular matrix remodeling in transgenic mice overexpressing tumor necrosis factor $\alpha$ can be modulated by anti-tumor necrosis factor $\alpha$ therapy. Proceedings of the National Academy of Sciences, v.97, n.23, p.12746-12751, 2000.

LI, P.G.; XU, J.W.; IKEDA, K. et al. Caffeic acid inhibits vascular smooth muscle cell proliferation induced by angiotensin II in stroke-prone spontaneously hypertensive rats. Hypertension Research, v.28, p.369-377, 2005.

LIBERA, L.D.; SABBADINI, R.; RENKEN, C. et al. Apoptosis in the skeletal muscle of rats with heart failure is associated with increased serum levels of the TNF $-\alpha$ and sphingosine. Journal of Molecular and Cellular Cardiology, v.33, p.1871-1878, 2001.

MARSHALL, R.P.; GOHLKE, P.; CHAMBERS, R.C. et al. Angiotensin II and the fibroproliferative response to acute lung injury. American Journal of Physiology Lung Cellular and Molecular Physiology, v.286, p.56-64, 2004.

MASCARENO, E.; BECKLES, D.L.; SIDDIQUI, M.A.. Janus kinase-2 signaling mediates apoptosis in rat cardiomyocytes. Vascular Pharmacology, v.43, n.5, p.327-335, 2005.
MOREAU, P.; D'USCIO, L.V.; SHAW, S. et al. Angiotensin II increases tissue endothelin and induces vascular hypertrophy - reversal by ETa receptor antagonist. Circulation, v.96, p.15931597, 1997.

MANN, D.L. Stress-activated cytokines and the heart: from adaptation to maladaptation. Annual Review of Physiology, v.65, p.81-101, 2003.

MANN, D.L. Targeted anticytokine therapy and the failing heart. American Journal of Cardiology, v.95, suppl 11A, p.9C-16C, 2005.

McMURRAY, J.J.; OSTERGREN, J.; SWEDBERG, $K$. et al. Effects of candesartan in patients with chronic heart failure and reduced left-ventricular systolic function taking angiotensin-convertingenzyme inhibitors: the CHARM-Added trial. Lancet, v.362, p.767-771, 2003.

NISHIMURA, H. Angiotensin receptors evolutionary overview and perspectives. Comparative Biochemistry and Physiology, v.128, p.11-30, 2001.

OKOSHI, M.P.; MATSUBARA, L.S.; FRANCO, M. et al. Myocyte necrosis is the basis for fibrosis in renovascular hypertensive rats. Brazilian Journal Medical and Biological Research, v.30, n.9, p.1135-1144, 1997.

PFEFFER, M.A.; MCMURRAY, J.J.; VELAZQUEZ, E.J. et al. Valsartan, captopril or both in myocardial infarction complicated by heart failure, left ventricular dysfunction or both. New England of Journal Medicine, v.349, p.1893-1906, 2003.

RAJAGOPAL, K.; LEFKOWITZ, R.J.; ROCKMAN, H.A. When 7 transmembrane receptors are not $\mathrm{G}$ protein coupled receptors. Journal of Clinical Investigation, v.15, n.11, p.2971-2974, 2005.

RAJAGOPALAN, S.; LAURSEN, J.B.;

BORTHAYRE, A. et al. Role for endothelin-1 in a agiotensin II-mediated hypertension. Hypertension, v.30, p.29-34, 1997.

SAMPAIO, W.O.; SOUZA DOS SANTOS, R.A.; FARIA-SILVA, R. et al. Angiotensin-(1-7) through receptor Mas mediates endothelial nitric oxide synthase activation via Akt-dependent pathways. Hypertension, v.49, n.1, p.185-192, 2007.

SANTOS, R.A.S.; FERREIRA, A.J. Angiotensin-(17) and the renin-angiotensin system. Current Opinion in Nephrology and Hypertension, v.16, p.122-128, 2007.

SANTOS, R.A.S.; SIMOES E SILVA, A.C.; MARIC, C. et al. Angiotensin-(1-7) is an endogenous ligand for the $G$ protein-coupled receptor Mas.

Proceedings of the National Academy of

Science, v.100, n.14, p.8258-8263, 2003 
SILVERTHORN, D.U. Fisiologia humana: uma abordagem integrada. 2. ed. São Paulo: Manole, 2003. Cap.19, p.581-583.

SUTTON, M.G.S.; SHARPE, N. Left ventricular remodeling after myocardial infarction pathophysiology and therapy. Circulation, v.101, p.2981-2988, 2000.

TORRE-AMIONE, G.; KAPADIA, S.; LEE, J. et al. Tumor necrosis factor- $\alpha$ and tumor necrosis factor receptors in the failing human heart. Circulation, v.93, p.704-711, 1996.

TANIMURA, M.; SHAROV, V.G.; SHIMOYAMA, $H$. et al. Effects of AT1-receptor blockade on progression of left ventricular dysfunction in dogs with heart failure. American Journal of Physiology, v.276, n.4, part 2, p.H1385-92, 1999.

VAILATI, M.C.F. Alterações histológicas do miocárdio, rim e fígado de ratos (Rattus novergicus) Wistar submetidos à infusão de angiotensina II. Influência na função ventricular esquerda e resposta ao tratamento com losartan e carvedilol. 2001. 110p. Tese (Doutorado). Faculdade de Medicina, Universidade Estadual Paulista, Botucatu.
VICKERS, C.; HALES, P.; KAUSHIK, V. et al. Hydrolysis of biological peptides by human angiotensin-converting enzyme-related carboxypeptidase. Journal of Biological Chemistry, v.277, p.14838-14843, 2002.

WEBER, KT. From inflammation to fibrosis: a stiff stretch of highway. Hypertension, v.43, p.716-719, 2004.

WU, R.; LAPLANTE, M.A.; DE CHAMPLAIN, J. Cyclooxygenase-2 inhibitors attenuate angiotensin II-induced oxidative stress, hypertension, and cardiac hypertrophy in rats. Hypertension, v.45, n.6, p.1139-1144, 2005.

ZIMMERMAN, M.; LAZARTIGUES, E.; SHARMA, R.V. et al. Hypertension caused by angiotensin II infusion involves increased superoxide production in the central nervous system. Circulation Research, v.95, p.210-216, 2004.

ZISMAN, L.S. ACE and ACE2: a tale of two enzymes. European Heart Journal, v.26, p.322324, 2005. 